\title{
Is completion lobectomy merited for unanticipated nodal metastases after radical segmentectomy for cT1 NO M0/pN1-2 non-small cell lung cancer?
}

\author{
Hiroaki Nomori, MD, PhD, ${ }^{\text {a }}$ Takeshi Mori, MD, PhD, ${ }^{\mathrm{b}}$ Yotaro Izumi, MD, $\mathrm{PhD},{ }^{\mathrm{a}}$ \\ Mitsutomo Kohno, $\mathrm{MD}, \mathrm{PhD},{ }^{\mathrm{a}}$ Kentaro Yoshimoto, $\mathrm{MD}, \mathrm{PhD},{ }^{\mathrm{b}}$ and Makoto Suzuki, MD, $\mathrm{PhD}^{\mathrm{b}}$
}

\begin{abstract}
Objective: To examine the role of radical segmentectomy, defined as a segmentectomy with extensive hilar/ mediastinal lymph node dissection and a sufficient surgical margin, for local control in cT1 N0 M0/pN1-2 non-small cell lung cancer (NSCLC), we examined the following: (1) whether metastases were observed in specimens additionally resected by completion lobectomy undertaken after segmentectomy because of pN1-2 disease and (2) prognostic outcome in patients whose operations were completed with segmentectomy regardless of pN1-2.
\end{abstract}

\begin{abstract}
Methods: Of 275 patients with cT1 N0 M0 NSCLC who were scheduled to undergo radical segmentectomy, $15(6 \%)$ had a diagnosis of $\mathrm{pN} 1$ or $\mathrm{N} 2$ disease. Of these patients, 10 were additionally treated with completion lobectomy, whereas the operations of the remaining 5 were completed with segmentectomy.
\end{abstract}
Results: None of the 10 patients who underwent completion lobectomy showed residual metastases in the spec- imens additionally resected by completion lobectomy. Two of the 5 patients whose operations were completed with segmentectomy, regardless of $\mathrm{N} 1$ or $\mathrm{N} 2$ disease, had tumor recurrence, but their first recurrence was not local.

Conclusions: Radical segmentectomy, with extensive hilar/mediastinal lymph node dissection and a sufficient surgical margin, may play a role in local control in patients with cT1 N0 M0/pN1-2 NSCLC. (J Thorac Cardiovasc Surg 2012;143:820-4)

In 1995, the Lung Cancer Study Group reported results of a prospective randomized controlled trial that assessed limited resection versus lobectomy for clinical T1 N0 M0 nonsmall cell lung cancer (NSCLC), concluding that the former was inferior to the latter because of greater local recurrence and poorer survival. ${ }^{1}$ Local recurrences after segmentectomy are known to be caused by the following factors: (1) insufficient surgical margin, (2) missed intrapulmonary metastases within the same lobe, and (3) missed lymph node metastases at the hilum and/or mediastinum. To avoid overlooking lymph node metastases, we submitted dissected lymph nodes for intraoperative examination to confirm the intraoperative N staging to be N0. However, we are usually worried about local recurrence at regional lymph nodes after segmentectomy, even if the intraoperative $\mathrm{N}$ staging was NO, because of the following: (1) intraoperative frozen

\footnotetext{
From the Department of General Thoracic Surgery, ${ }^{\text {a }}$ School of Medicine, Keio University, Tokyo, Japan; and Department of Thoracic Surgery, ${ }^{b}$ Graduate School of Medical Sciences, Kumamoto University, Kunamoto, Japan.

Disclosures: Authors have nothing to disclose with regard to commercial support.

Received for publication July 10, 2011 ; revisions received Sept 13, 2011; accepted for publication Oct 20, 2011; available ahead of print Nov 21, 2011.

Address for reprints: Hiroaki Nomori, MD, PhD, Division of General Thoracic Surgery, Department of Surgery, School of Medicine, Keio University, 35 Shinanomachi, Shinjuku-ku, 160-8582, Tokyo, Japan (E-mail: hnomori@qk9.so-net.ne.jp). $0022-5223 / \$ 36.00$

Copyright ( $) 2012$ by The American Association for Thoracic Surgery doi:10.1016/j.jtcvs.2011.10.045
}

section diagnosis cannot always recognize metastases in all of the lymph nodes and (2) the dissection of intrapulmonary lymph nodes remaining within the preserved segment is impossible.

Since April 2005, we have been conducting radical segmentectomy, defined as a segmentectomy with hilar/mediastinal lymph node dissection and taking a sufficient surgical margin in patients with cT1 N0 M0 NSCLC. ${ }^{2}$ For accurate intraoperative $\mathrm{N}$ staging, we usually identify sentinel nodes (SNs) after dissection of hilar/mediastinal lymph nodes, including segmental nodes of the preserved segments. ${ }^{3}$ When intraoperative frozen sections of the SNs show metastasis, we usually perform completion lobectomy. In the present study, to examine the role of segmentectomy with this systematic lymph node dissection as a local control in patients with cT1 N0 M0 NSCLC in whom pN1 or N2 disease was finally diagnosed, we examined whether lymph node metastasis remained within the specimens additionally resected by completion lobectomy. We also examined the prognostic outcome of patients with cT1 N0 M0 whose operations were completed with segmentectomy regardless of $\mathrm{pN} 1$ or N2 stage.

\section{PATIENTS AND METHODS Eligibility}

The study protocol for segmentectomy using radioisotopes for $\mathrm{SN}$ identification in patients with cT1 N0 M0 NSCLC was approved by the ethics 


\section{Abbreviations and Acronyms \\ NSCLC $=$ non-small cell lung cancer \\ $\mathrm{SN} \quad=$ sentinel node}

committees of Kumamoto University and Keio University. The criteria for segmentectomy in the study were as follows: (1) peripheral type cT1 N0 MO NSCLC; (2) exclusion of tumors of the right middle lobe; (3) no evidence of metastasis on intraoperative frozen sections of lymph nodes; (4) surgical margin more than $2 \mathrm{~cm}$ or longer than tumor size; (5) normal serum level of carcinoembryonic antigen; and (6) segmentectomy requested by patients. Clinical staging was performed by body computed tomographic scanning, brain magnetic resonance imaging, and fluorodeoxyglucose positron emission tomography in all patients. Lymph node biopsy via endobronchial ultrasonography was conducted for swelling lymph nodes, whereas mediastinoscopy was not undertaken. All patients provided informed consent after fully discussing the risks and benefits of segmentectomy with their surgeons.

\section{Patients}

From April 2005 to March 2009, 247 patients with cT1 N0 M0 NSCLC underwent surgical treatment at Kumamoto University Hospital. From April 2009 to March 2011, 130 patients with cT1 N0 M0 underwent surgical treatment at Keio University Hospital. Segmentectomy was planned in $275(73 \%)$ of the total 377 patients, lobectomy in $59(16 \%)$, and wedge resection in $43(11 \%)$. A corresponding author (H.N.) participated in all of these segmentectomies. The reasons for conducting lobectomy in the 59 patients were tumors existed in the right middle lobe in 27 patients, centrally located tumors in 10 , multiple lesions in the same lobe in 9 , high carcinoembryonic antigen level in 7, patient's request for lobectomy in 5, and severe emphysematous change of the tumor-existing lobe in the remaining 1. Of the 275 patients who were scheduled for segmentectomy, $205(75 \%)$ underwent SN identification using ${ }^{99 m}$ technetium-tin colloid or ${ }^{99 m}$ technetium-phytate to target lymph nodes submitted for intraoperative frozen section diagnosis, as reported before, ${ }^{4-7}$ whereas the remaining $70(25 \%)$ did not. The nomenclature used for pulmonary segments is shown in Table 1.

\section{Operative Procedures and Lymph Node Evaluation}

Although the SNs were identified in the present study, the purpose of this study was to target lymph nodes submitted for intraoperative frozen section but not to reduce lymph node dissection. Under thoracotomy, segmentectomy with lymph node dissection was performed as reported previously. ${ }^{3}$ After resection of the affected segment, lymph node stations 10 to 13 , including station 13 of preserved segments, were dissected. Lymph nodes at both the superior and inferior mediastinum were dissected, regardless of tumor location. The identified SNs were submitted for intraoperative frozen section diagnosis. They were serially cut to about $3 \mathrm{~mm}$ in thickness followed by hematoxylin and eosin staining to detect even a small metastasis. If the intraoperative frozen sections of SNs showed no metastasis, the operation was completed with segmentectomy. If the frozen sections of SNs showed metastasis, completion lobectomy was performed. SNs were further examined immunohistochemically after surgery by using anticytokeratin AE1/AE3 antibody (Dako Corporation, Carpinteria, Calif) to identify possible microscopic metastases. For patients in whom SN identification was not conducted, at least the No. 12, No. 13, and lobespecific mediastinal nodes were submitted for frozen sections. Lymph node nomenclature and tumor staging were based on the 2009 Staging Manual in Thoracic Oncology of the International Association for the Study of Lung Cancer (Table 2). ${ }^{8}$ During the operation, the surgical margin was confirmed to be sufficiently longer than $2 \mathrm{~cm}$ or the maximum diameter of the tumor.
TABLE 1. Pulmonary segment nomenclature

\begin{tabular}{cl}
\hline Lobe & \\
Right upper lobe & \\
S1 & Apical \\
S2 & Dorsal \\
S3 & Ventral \\
Left upper lobe & \\
S1+2 & Apicodorsal \\
S3 & Ventral \\
S4 & Lingular superior \\
S5 & Lingular inferior \\
Lower lobe & \\
S6 & Superior \\
S7 & Mediobasal \\
S8 & Ventrobasal \\
S9 & Laterobasal \\
S10 & Dorsobasal \\
\hline
\end{tabular}

\section{Pathologic Examination of Specimens Resected by Completion Lobectomy}

The specimens additionally resected by completion lobectomy, that is, the resection of the preserved lobe after segmentectomy, were pathologically examined. The lymph nodes within the additionally resected specimens were also examined by immunohistochemical staining using anticytokeratin antibody.

\section{Postoperative Follow-up}

All patients were followed up every 3 to 6 months after surgery until 5 years after surgery and yearly thereafter. The follow-up period ranged from 6 to 73 months, both of which average and median periods were 41 months. Patients with pN1 or N2 were usually treated by chemotherapy after surgery with platinum doublet.

\section{RESULTS}

Lymph node metastases were found in $15(6 \%)$ of the 275 patients who were scheduled for segmentectomy. The surgical margin was free of tumor in all of the 15 patients. Of the 15 patients with lymph node metastases, 10 underwent completion lobectomy after segmentectomy (Table 3). All of these 10 patients showed metastases in SNs. Patients 5, 6, and 8 underwent completion lobectomy

TABLE 2. Lymph node nomenclature

\begin{tabular}{|c|c|c|c|}
\hline \multicolumn{2}{|r|}{ N2 node } & \multicolumn{2}{|c|}{ N1 node } \\
\hline \multicolumn{2}{|c|}{ Superior mediastinal } & \multicolumn{2}{|l|}{ Hilar } \\
\hline \#2 & Upper paratracheal & $\# 10$ & Hilar \\
\hline \#3 & Prevascular and retrotracheal & \#11 & Interlobar \\
\hline \#4 & Lower paratracheal & \#12 & Lobar \\
\hline \#5 & Subaortic & \multicolumn{2}{|c|}{ Intrapulmonary } \\
\hline \multirow[t]{2}{*}{$\# 6$} & Para-aortic & \#13 & Segmental \\
\hline & & \#14 & Subsegmental \\
\hline \multicolumn{4}{|c|}{ Inferior mediastinal } \\
\hline \#7 & Subcarinal & & \\
\hline \#8 & Paraesophageal & & \\
\hline \#9 & Pulmonary ligament & & \\
\hline
\end{tabular}


TABLE 3. Patients underwent completion lobectomy after segmentectomy because of N1 or N2 disease

\begin{tabular}{|c|c|c|c|c|c|c|c|c|c|}
\hline \multirow[b]{2}{*}{ No. (age/sex) } & \multirow[b]{2}{*}{ cTNM } & \multicolumn{2}{|c|}{ Tumor } & \multirow[b]{2}{*}{ LN metastasis } & \multirow[b]{2}{*}{ Segmentectomy } & \multirow{2}{*}{$\begin{array}{l}\text { Completion } \\
\text { lobectomy }\end{array}$} & \multirow[b]{2}{*}{ pTNM } & \multirow{2}{*}{$\begin{array}{c}\text { First } \\
\text { recurrence site }\end{array}$} & \multirow[b]{2}{*}{ Outcome } \\
\hline & & Histology & Size $(\mathrm{cm})$ & & & & & & \\
\hline $1(50 / \mathrm{F})$ & T1b N0 M0 & Ad & 2.7 & $\# 5,6,10,12$ & 1t. S1-3 & LUL & T1a N2 M0 & Brain & $38 \mathrm{mo}$, dead \\
\hline $2(72 / \mathrm{M})$ & T1a N0 M0 & Ad & 2.0 & \#11 & rt. S6 & RLL & T1a N1 M0 & Pleura & $24 \mathrm{mo}$, dead \\
\hline $3(54 / F)$ & T1b N0 M0 & Ad & 2.4 & $\# 7,12$ & lt. $S 9+10$ & LLL & $\mathrm{T} 2 \mathrm{~N} 2 \mathrm{M} 0$ & - & $67 \mathrm{mo}$, alive \\
\hline $4(73 / \mathrm{M})$ & T1a N0 M0 & $\mathrm{Sq}$ & 2.0 & \#5 & lt. $\mathrm{S} 1+2$ & LUL & T1a N2 M0 & - & 39 mo, alive \\
\hline $5(35 / F)$ & T1b N0 M0 & Ad & 2.2 & \#12 & lt. S6 & LLL & T1b N1 M0 & - & $20 \mathrm{mo}$, alive \\
\hline $6(38 / \mathrm{F})$ & T1b N0 M0 & Ad & 2.3 & $\# 12$ & lt. $S 1-3$ & LUL & T1b N1 M0 & - & 19 mo, alive \\
\hline $7(58 / \mathrm{M})$ & T1a N0 M0 & Ad & 1.3 & $\# 4$ & rt. S1 & RUL & T1a N2 M0 & - & $10 \mathrm{mo}$, alive \\
\hline $8(47 / F)$ & T1a N0 M0 & Ad & 1.6 & $\# 13$ & 1t. $S 9+10$ & LLL & T1a N1 M0 & - & 9 mo, alive \\
\hline $9(63 / \mathrm{M})$ & T1b N0 M0 & Ad & 2.1 & $\# 12$ & rt. S8 & RLL & T3 N2 M0 & - & 7 mo, alive \\
\hline $10(51 / \mathrm{M})$ & T1a N0 M0 & Ad & 2.0 & \#4 & rt. S9 & RLL & T1a N2 M0 & - & $6 \mathrm{mo}$, alive \\
\hline
\end{tabular}

$L N$, Lymph node; $A d$, adenocarcinoma; $S q$, squamous cell carcinoma; rt., right; lt., left; $L U L$, left upper lobe; $R L L$, right lower lobe; $L L L$, left lower lobe; $R U L$, right upper lobe.

a few days after segmentectomy, because immunohistochemical staining on deep cut section of the SN after surgery showed micrometastasis, whereas intraoperative frozen sections showed no metastasis by hematoxylin and eosin staining. In none of these 10 patients did specimens additionally resected by completion lobectomy show residual tumors in the lungs or lymph nodes, even with immunohistochemical staining. Whereas patients 1 and 2 died of the disease, the remaining 8 patients are alive without disease through July 2011.

The remaining 5 patients had their operations completed by segmentectomy regardless of $\mathrm{pN} 1$ or $\mathrm{N} 2$ disease (Table 4), for the following reasons: (1) old age in patients 11 and 12 ; (2) patient's request in patient 13 ; (3) central-type squamous cell carcinoma at the segmental bronchus in patient 14 with poor pulmonary function, which was treated with sleeve segmentectomy; and (4) double lung cancers in patient 15 , both of which were resected by segmentectomy. Although patients 11 and 12 died of the disease, their first recurrence sites were distant organs. The other 3 patients are alive without disease 6, 9, 28 months after surgery, respectively.

Skip metastases to the mediastinal lymph nodes were found in $4(27 \%)$ of the 15 patients: patients $4,7,10$, and 12. Of these, the patient with adenocarcinoma in the right lower lobe (patient 10) had single metastasis at the \#4R, which was not a lobe-specific mediastinal node.

\section{COMMENTS}

The present results highlight the following 3 points: (1) the specimens additionally resected by completion lobectomy did not show any residual metastases even with immunohistochemical staining in the 10 patients with $\mathrm{pN} 1$ or $\mathrm{N} 2$ disease; (2) although 2 of the 5 patients who had their operations completed by segmentectomy regardless of $\mathrm{pN} 1$ or $\mathrm{N} 2$ disease died of the disease, their first recurrence sites were not local; and (3) whereas the follow-up period is still not sufficient, the remaining 3 of the 5 patients who had their operations completed by segmentectomy regardless of $\mathrm{N} 1$ or $\mathrm{N} 2$ have been alive without recurrences. These results indicate that segmentectomy may play a role in local control in patients with cT1 N0 M0/pN1-2 NSCLC if combined with systematic lymph node dissection of the hilum and mediastinum and sufficient surgical margin.

Skip metastasis to the mediastinal lymph nodes has been reported to occur in $20 \%$ to $40 \%$ of patients with NSCLC, ${ }^{9}$ which may be caused by some lymphatic flow going directly to the mediastinum rather than to the hilum. Four $(27 \%)$ of the present 15 patients also showed skip metastasis to the mediastinal lymph nodes. Therefore, mediastinal lymph node dissection is necessary during segmentectomy even in patients with cT1 N0 M0 NSCLC. Although the American College of Surgeons Oncology Group Z0030 randomized controlled trial between mediastinal lymph node dissection versus sampling showed no difference, ${ }^{10}$ we

TABLE 4. Patients whose operations were completed with segmentectomy regardless of N1 or N2 disease

\begin{tabular}{|c|c|c|c|c|c|c|c|c|}
\hline \multirow[b]{2}{*}{ No. (age/sex) } & \multicolumn{2}{|c|}{ Tumor } & \multirow[b]{2}{*}{ Size $(\mathbf{c m})$} & \multirow[b]{2}{*}{ LN metastasis } & \multirow[b]{2}{*}{ Segmentectomy } & \multirow[b]{2}{*}{ pTNM } & \multirow[b]{2}{*}{ First recurrence } & \multirow[b]{2}{*}{ Outcome } \\
\hline & cTNM & Histology & & & & & & \\
\hline $11(80 / \mathrm{M})$ & T1b N0 M0 & Ad & 2.5 & $\# 5,12,13$ & 1t. $\mathrm{S} 1-3$ & T1b N2 M0 & Intestine + mediastinum & $20 \mathrm{mo}$, dead \\
\hline $12(81 / \mathrm{M})$ & T1a N0 M0 & Ad & 1.0 & $\# 4$ & lt. $\mathrm{S} 4+5$ & T1a N2 M0 & Liver & $18 \mathrm{mo}$, dead \\
\hline $13(68 / F)$ & T1a N0 M0 & Ad & 1.2 & $\# 4,12,13$ & 1t. S8 & T1a N2 M0 & - & $27 \mathrm{mo}$, alive \\
\hline $14(75 / \mathrm{M})$ & T1a N0 M0 & $\mathrm{Sq}$ & 1.5 & \#13 & 1t. $\mathrm{S} 1+2$ & T1a N1 M0 & - & 9 mo, alive \\
\hline \multirow[t]{2}{*}{$15(62 / \mathrm{M})$} & T1b N0 M0 & $\mathrm{Ad}^{*}$ & 2.3 & \#13 & 1t. S1-3 & T1b N1 M0 & - & 6 mo, alive \\
\hline & T1a N0 M0 & $\mathrm{Ad}^{*}$ & 1.5 & - & 1t. S6 & T2a N0 M0 & & \\
\hline
\end{tabular}

$L N$, Lymph node; $A d$, adenocarcinoma; $S q$, squamous cell carcinoma; 1 ., left. *Double lung cancers. 
did the extensive lymph node dissection in this study to verify whether the segmentectomy itself was a radical procedure for cT1 N0 M0 NSCLC. In the future, segmentectomy with lymph node sampling should be further evaluated as a radical treatment or not.

It has been reported that metastasis of mediastinal lymph nodes is mostly lobe-specific, that is, upper mediastinal lymph nodes in tumors of the upper lobe and lower mediastinal lymph nodes in those of the lower lobe. ${ }^{9,11}$ However, 1 of the present patients, who had primary tumors in the right lower lobe, showed metastasis only at the upper mediastinal node, that is, \#4R, which was not lobe-specific. $\mathrm{We}^{6}$ previously reported that the SNs of NSCLCs in the right lower lobe were found in \#4R at a rate of $36 \%$. We therefore believe that \#4R should be the target of lymph node sampling for cT1 N0 M0 NSCLCs of the right lower lobe, not only during segmentectomy but also during lobectomy.

The final indication for segmentectomy in most of the present patients $(75 \%)$ was determined by intraoperative frozen section diagnosis of SNs identified by the radioisotope method. However, we consider that SN identification is not always necessary for intraoperative $\mathrm{N}$ staging during segmentectomy. In its place, if SN identification is not used during segmentectomy, all of the dissected lymph nodes should be submitted theoretically for frozen section diagnosis, because the frequency of lymph node metastasis in cT1 NO MO NSCLC is reported to be about $10 \%$ as reported in the recent Japanese Lung Cancer Registry Study. ${ }^{12}$ However, intraoperative frozen section diagnosis of all dissected lymph nodes is not feasible. Therefore, when not using SN identification, we usually target lymph nodes submitted for frozen section diagnosis during segmentectomy as follows: (1) hilar lymph nodes 12 and 13 are submitted for frozen section rather than nodes 10 or 11 , because our previous study showed that $\mathrm{SN}$ rates for nodes 12 and 13 were $39 \%$ and $57 \%$, respectively, whereas those for nodes 10 and 11 were only $10 \%$ and $16 \%$, respectively ${ }^{5}$; (2) in mediastinal lymph nodes, the largest lymph node in each station is submitted for frozen section, because they had almost $90 \%$ of the rate of having metastasis in N2 disease according to our previous report ${ }^{13}$; and (3) although lobespecific mediastinal nodes should be targeted, the \#4R is also submitted for intraoperative frozen section in NSCLC of the right lower lobe, on the basis of our previous findings. ${ }^{6}$

Whereas several reports have shown that segmentectomy provides a similar prognosis as lobectomy for T1 N0 M0 NSCLC, ${ }^{14-16}$ our study presents the possibility of radical segmentectomy as a local control even for cT1 N0 M0/ pN1-2 NSCLC. The radical segmentectomy described in the present study should be distinguished from a simple segmentectomy on the basis of the extent of lymph node dissection. It seems similar to the "radical lobectomy" proposed by Cahan ${ }^{17}$ in 1960, when pneumonectomy was a standard procedure. Cahan defined a radical lobectomy as an operation in which 1 or 2 lobes were excised in a block dissection with their regional hilar and mediastinal lymph nodes. After his proposal, lobectomy with mediastinal lymph node dissection replaced pneumonectomy even for N1 or N2 disease, and this technique has been established as a standard procedure for NSCLC to date. However, even after radical lobectomy, several studies have reported the development of local recurrences, at a rate of $6 \%{ }^{1,18} \mathrm{We}$ therefore consider that that local recurrence after radical segmentectomy may be acceptable at a rate of up to $6 \%$; in contrast to this hypothesis, the present study showed neither local recurrence after segmentectomy nor tumors within the specimens additionally resected by completion lobectomy.

Even by using extensive lymph node dissection and taking a sufficient surgical margin during segmentectomy, the procedure may miss pulmonary metastases within the same lobe. However, the recently developed high-resolution computed tomography can identify pulmonary lesions even just $0.3 \mathrm{~cm}$ in size, which can lead to a correct indication for segmentectomy.

Here, we present pathologic results of specimens additionally resected by completion lobectomy in patients with cT1 N0 M0/pN1-2 NSCLC and the prognostic outcome of patients whose operations were completed by segmentectomy regardless of $\mathrm{pN} 1$ or $\mathrm{N} 2$ disease. On the basis of our promising findings, we could not find any merit of completion lobectomy after radical segmentectomy. We believe that radical segmentectomy, with extensive hilar/mediastinal lymph node dissection and taking a sufficient surgical margin, may play as much of a role in local control as lobectomy in patients with cT1 N0 M0/pN1-2 NSCLC.

\section{References}

1. Ginsberg RH, Rubinstein LV. Randomized trial of lobectomy versus limited resection for T1 N0 non-small cell lung cancer: Lung Cancer Study Group. Ann Thorac Surg. 1995;60:615-23.

2. Nomori H, Ikeda K, Mori T, Kobayashi H, Iwatani K, Kawanaka K, et al. Sentinel node navigation segmentectomy for clinical stage IA non-small cell lung cancer. J Thorac Cardiovasc Surg. 2007;133:780-5.

3. Nomori H, Ohba Y, Shibata H, Shiraishi K, Mori T, Shiraishi S. Required area of lymph node sampling during segmentectomy for clinical stage IA non-small cell lung cancer. J Thorac Cardiovasc Surg. 2010;139:38-42.

4. Nomori H, Horio H, Naruke T, Orikasa H, Yamazaki K, Suemasu K. Use of technetium-99m tin colloid for sentinel lymph node identification in non-small cell lung cancer. J Thorac Cardiovasc Surg. 2002;124:486-92.

5. Nomori H, Watanabe K, Ohtsuka T, Naruke T, Suemasu K. In vivo identification of sentinel nodes for clinical stage I non-small cell lung cancer for abbreviation of mediastinal lymph node dissection. Lung Cancer. 2004;46:49-55.

6. Nomori H, Ikeda K, Mori T, Shiraishi S, Kobayashi H, Iwatani K, et al. Sentinel node identification in clinical stage Ia non-small cell lung cancer by a combined single photon emission computed tomography/computed tomography system. J Thorac Cardiovasc Surg. 2007;134:182-7.

7. Nomori H, Ohba Y, Yoshimoto K, Shibata H, Mori T, Shiraishi S, et al. Difference of sentinel lymph node identification between tin colloid and phytate in patients with non-small cell lung cancer. Ann Thorac Surg. 2009;87: 906-10

8. Goldstraw P, executive editor. Staging manual in thoracic oncology. International Association for the Study of Lung Cancer. San Francisco: 2009. 
9. Naruke T, Tsuchiya R, Kondo H, Nakayama H, Asamura H. Lymph node sampling in lung cancer: how should it be done? Eur J Cardiothorac Surg. 1999;16:S17-24.

10. Scott WJ, Allen MS, Darling G, Meyers B, Decker PA, Putnam JB, et al. Videoassisted thoracic surgery versus open lobectomy for lung cancer: a secondary analysis of data from the American College of Surgeons Oncololgy Group Z0030 randomized clinical trial. J Thorac Cardiovasc Surg. 2010;139:976-83.

11. Okada M, Tsubota N, Yoshimura M, Miyamoto Y, Matsuoka H. Prognosis of completely resected pN2 non-small cell lung carcinomas: what is the significant node that affects survival? J Thorac Cardiovasc Surg. 1999;118:270-5.

12. Sawabata N, Miyaoka E, Asamura H, Nakanishi Y, Eguchi K, Mori M, et al. Japanese Lung Cancer Registry Study of 11,663 surgical cases in 2004: demographic and prognosis changes over decade. J Thorac Oncol. 2011;6:1229-35.

13. Ikeda K, Nomori H, Mori T, Kobayashi H, Iwatani K, Yoshimoto K. Size of metastatic and non-metastatic mediastinal lymph nodes in non-small cell lung cancer. J Thorac Oncol. 2006;1:949-52.
14. Tsubota N, Ayabe K, Doi O, Mori T, Namikawa S, Taki T, et al. Ongoing prospective study of segmentectomy for small lung tumors. Study Group of Extended Segmentectomy for Small Lung Tumor. Ann Thorac Surg. 1998; 66:1787-90.

15. Kodama K, Doi O, Higashiyama M, Yokouchi H. Intentional limited resection for selected patients with T1 N0 M0 non-small cell lung cancer: a single-institution study. J Thorac Cardiovasc Surg. 1997;114:347-53.

16. Okada M, Koike T, Higashiyama M, Yamato Y, Kodama K, Tsubota N. Radical sublobar resection for small-sized non-small cell lung cancer: a multicenter study. J Thorac Cardiovasc Surg. 2006;132:769-75.

17. Cahan WG. Radical lobectomy. J Thorac Cardiovasc Surg. 1960;39:555-72.

18. Varlotto JM, Recht A, Flickinger JC, Medford-Davis LN, Dyer AM, DeCamp MM. Varying recurrence rates and risk factors associated with different definitions of local recurrence in patients with surgically resected, stage I nonsmall cell lung cancer. Cancer. 2010;116:2390-400.

Access to The Journal of Thoracic and Cardiovascular Surgery Online is reserved for print subscribers!

Full-text access to The Journal of Thoracic and Cardiovascular Surgery Online is available for all print subscribers. To activate your individual online subscription, please visit The Journal of Thoracic and Cardiovascular Surgery Online, point your browser to http://www.mosby.com/itcvs, follow the prompts to activate your online access, and follow the instructions. To activate your account, you will need your subscriber account number, which you can find on your mailing label (note: the number of digits in your subscriber account number varies from 6 to 10 ). See the example below in which the subscriber account number has been circled:

\section{Sample mailing label}

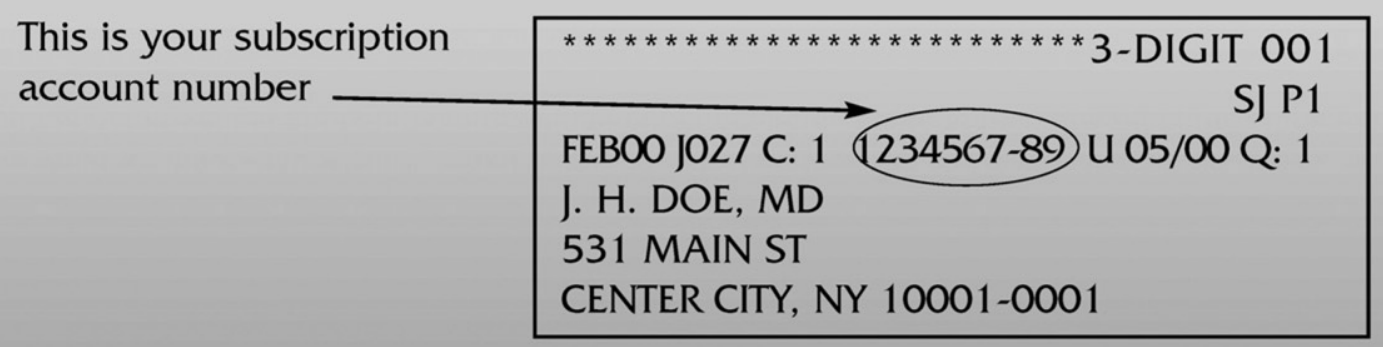

Personal subscriptions to The Journal of Thoracic and Cardiovascular Surgery Online are for individual use only and may not be transferred. Use of The Journal of Thoracic and Cardiovascular Surgery Online is subject to agreement to the terms and conditions as indicated online. 